\title{
A Hybrid Image Denoising Technique Using Neighbouring Wavelet Coefficients
}

\author{
Mantosh Biswas ${ }^{1}$ and Hari Om² \\ ${ }^{1}$ Department of Computer Engineering, NIT Kurukshetra, India \\ ${ }^{2}$ Department of Computer Science \& Engineering, IIT Dhanbad, India \\ Email: mantoshb@gmail.com, hari.om.cse@ismdhanbad.ac.in
}

\begin{abstract}
This paper proposes a hybrid image denoising technique using neighbouring wavelet coefficients. The NeighShrink method groups the wavelet coefficients in non overlapping blocks and then thresholds empirically them blockwise. This method does not give good quality of image since it removes too many small wavelet coefficients. Our proposed scheme retains the modified coefficients and also gives good performance in terms of peak signal-to-noise ratio.
\end{abstract}

Keywords: Wavelet thresholding, image denoising, neighbouring coefficients, peak signal to-noise.

\section{Introduction}

Wavelet $[1-2,16]$ transformations are common tools in various fields such as image compression, image denoising, signal processing and pattern recognition. In last decade and so, based on wavelet transforms several new methods have been emerged to remove the additive white Gaussian noise (AWGN) from the images [2]. Such image denoising procedures are also known as wavelet shrinkage or thresholding [3-4]. There exist various methods for wavelet thresholding that rely on the choice of a threshold value such as VisuShrink [5-6], NormalShrink [10], NeighShrink [11-13] and ModiNeighShrink [14].

VisuShrink was proposed by Donoho [5-7]. It does not deal with minimizing the mean squared error. Donoho and Johnstone have discussed SureShrink [7-8] method based on Stein's Unbiased Risk Estimator (SURE) that minimizes the mean squared error. SureShrink method uses the combination of universal threshold and SURE threshold. The employed thresholding in that method is adaptive in the sense that if the unknown function contains abrupt changes or boundaries in the image, the reconstructed image also keeps these changes. BayesShrink [9], proposed by Chang et al., is a datadriven adaptive image denoising method. Elyasi and Zarmehi have discussed NormalShrink [10] which is another data-driven image denoising method. NeighBlock [11-12] uses local block thresholding rules that were discussed by Chen et al. and Cai for wavelet function estimation. This method either kills or keeps all the coefficients in groups. Generally, the threshold level and block size are very crucial in the performance of a block thresholding estimator. The NeighShrink [13] outperforms over the SureShrink. Divya Guglani and Nitin Kumar Katyal [21] proposed denoising method based on NeighShrinkSure thresholding \& median filter and their performance is good over VisuShrink, SureShrink, and BayesShrink.

There has been a fair amount of research to select the threshold for image denoising from noisy image using wavelet $[15,17-18,20]$. The selection of a threshold is very important in noise removal in images because a denoising method most frequently produces smoothed images [4]. The challenge of denoising method is to reconstruct good quality of image from noisy one based on thresholding and shrinking function. The main issue is finding appropriate threshold and shrinkage function for removing the noise, as well as restoring wavelet coefficients from noisy image. Therefore our proposed works do it perfectly by considering thresholding and shrinking functions. Therefore, in this paper, we extend the idea of Cai and Silverman that is used for image denoising. The rest of the paper is organized as follows: Section 2 discusses the proposed work. Experimental results and discussions are given in section 3. Finally, the conclusion is given in section 4 . 


\section{Proposed Work}

The basic model of the corrupted image by white Gaussian noise is given by:

$$
G_{i, j}=X_{i, j}+h_{i, j}
$$

where $\mathrm{G}_{\mathrm{i}, \mathrm{j}}$ and $\mathrm{X}_{\mathrm{i}, \mathrm{j}}, 1 \leqslant \mathrm{i}, \mathrm{j} \leqslant \mathrm{M}$ represent corrupted image, and original image, respectively, of size $\mathrm{M} \times \mathrm{M}$. $\sigma$ is noise level which is identically distributed (i.i.d) zero mean, additive white Gaussian Noise $n_{i, j}$.

Let $\mathrm{W}$ and W-1 denote two dimensional orthogonal discrete wavelet transform (DWT) and its inverse (IDWT) matrices, respectively. On applying wavelet transform $\mathrm{W}$ on noisy image $\mathrm{G}$ we get wavelet coefficients of corrupted image G. This may be written as:

$$
Y=W G
$$

After applying threshold function on $\mathrm{Y}$, we get an image denoting it by $\bar{X}$. The denoised estimate, denoted by $\tilde{X}$, of the original image $\mathrm{X}$ is given by applying the inverse wavelet transform on $\bar{X}$, i.e.,

$$
\tilde{X}=W^{-1} \bar{X}
$$

We now discuss the parameter estimation. The VisuShrink [5-6] method is also known as Universal threshold. We denote threshold by $\mathrm{T} 1$ that is defined as follows:

$$
T 1=\sigma \sqrt{2 \log M}
$$

where $\mathrm{M}$ represents the signal size and $\sigma 2$ noise variance that is given by:

$$
\sigma^{2}=\left[\left(\text { median }\left|Y_{i j}\right|\right) / 0.6745\right]^{2}, Y_{i j} H H_{1}
$$

In our proposed method, we take the threshold function T2 of NormalShrink [10] method that is defined as follows:

$$
T 2=\beta \sigma 2 / \sigma y
$$

where $\sigma 2$ is the noise variance and $\sigma y$ is the standard deviation of the subband and $\beta$ is the scale parameter that is computed once for each scale using the following:

$$
\beta=\sqrt{\log \frac{L_{K}}{R}}
$$

Here Lk represents the subband length at kth scale and $R$ represents maximum number of decompositions.

The denoising method of NeighShrink and modified NeighShrink used threshold of Visushrink but in our work we used NormalShrink threshold function. In Visushrink threshold function it gives the fixed and larger value than NeighShrink threshold function, and the value is changing with decomposition level and it is not much more or less value Visushrink threshold value. Therefore accordingly larger threshold can't reconstruct and modify properly the wavelet coefficients in noisy image i.e., killing more wavelet coefficients, but our threshold value is neither large nor low and it changes with decomposition level, so our threshold can easily reconstruct and modify properly the noisy wavelet coefficients of the image and get good quality of noisy free image.

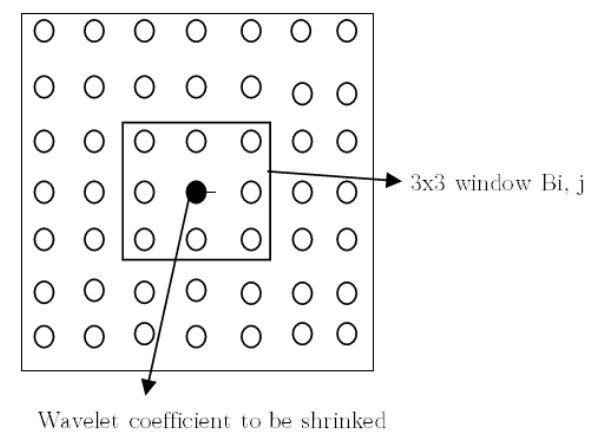

Figure 1. An illustration of the neighborhood window centered at the wavelet coefficient to be shrinked 
Besides, for NormalShrink's threshold, we also incorporate neighbouring coefficients in thresholding process that is given by Cai and Silverman [11]. For incorporating neighbouring coefficients, we consider a square window of odd number pixels in its length that is centered at the pixel to be thresholded as shown in Fig. 1 for window size of $3 \times 3$. Suppose di,j is the set of wavelet coefficients of the noisy signal in a window Bi,j. The wavelet coefficients of different subbands are thresholded independently.

Let $S_{i, j}^{2}$ denote the sum of square of the wavelet coefficients di,j in the neighborhood window Bi,j, i.e.,

$$
S_{i, j}^{2}=\sum_{(i, l) \in B_{i, j}} d_{i, l}^{2}
$$

Define $\beta_{i, j}$ in terms of $S_{i, j}^{2}$ as follows:

$$
\beta_{i, j}=\left(1-\frac{T_{2}^{2}}{S_{i, j}^{2}}\right)_{+}
$$

The sign "+" in this relation means to keep the positive value and discard the negative values, i.e., set it to zero for a negative value.

Now shrink the wavelet coefficients by $\beta_{i, j}$ as follows:

$$
\hat{d}_{i, j}=d_{i, j} \beta_{i, j}
$$

After applying this shrinkage factor, it has been observed that the reconstructed image has mat like aberrations when the noise content is high. Using NeighShrink, we lose some image details and sometimes the reconstructed image becomes blurred. The reason for this blurring may be the suppression of too many details of wavelet coefficients. This problem can be avoided using the following shrinkage factor [14].

$$
\beta_{i, j}=\left(1-\frac{3}{4} \frac{T_{2}^{2}}{S_{i, j}^{2}}\right)_{+}
$$

\subsection{Denoising Algorithm}

In the proposed scheme following steps are performed:

Transform noisy image into an orthogonal domain by 2-D discrete wavelet transform.

Apply thresholding to the resulting wavelet coefficients by using the threshold function T2 from (3).

Perform 2-D inverse discrete wavelet transform on the image after applying thresholding to obtain the denoised image $\tilde{X}$.

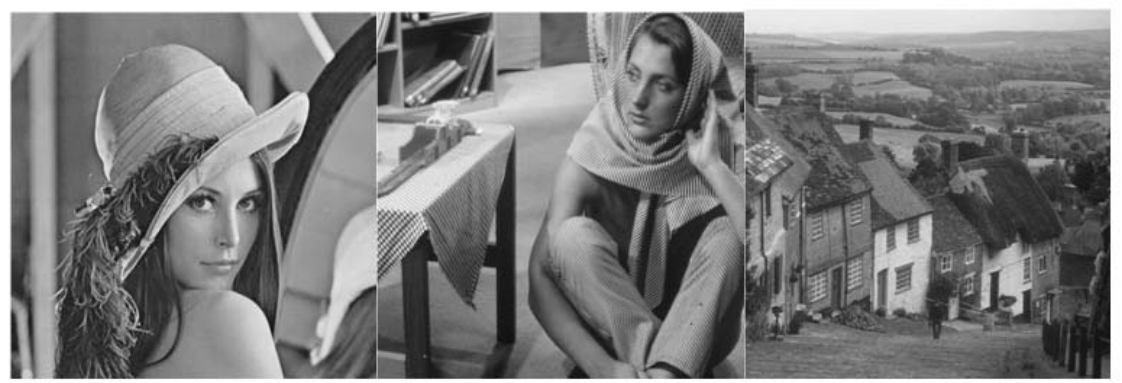

(a)

(b)

(c)

Figure 2. Original test images with $512 \times 512$ pixels: (a) Lena (b) Barbara and (c) Goldhill

\section{$3 \quad$ Results and Discussions}

We have performed experiments using NeighShrink and ModiNeighShrink schemes on several test images widely used in image processing community. In our discussion, three images namely Lena, 
Goldhill and Barbara of $512 \times 512$ size each (refer Fig. 2) have been taken with different noise levels, i.e., 10, 20, 30, 35, and 50 of additive Gaussian white noise. The Daubechies [19] wavelets with eight vanishing moments have been used with four decomposition scales. The experimental results have been evaluated using the quality measure i.e. PSNR which is calculated using the following formula:

$$
\operatorname{PSNR}(\text { in } d b)=10 \log 10(255) 2 / \mathrm{MSE}
$$

where, MSE is the mean squared error between the original and reconstructed images. The results are shown in Tables 1-3 for taking window sizes of 3x3, 5x5, and 7x7, respectively. It is evident from Tables 1-3 that our proposed technique outperforms over NeighShrink and ModiNeighShrink for window size $3 \times 3$ using different noise levels. In case of window size $5 \times 5$, our technique outperforms the ModiNeighShrink when the noise level is more than 10. Furthermore, it gives good results for window size $7 \times 7$ over the ModiNeighShrink when the noise level is more than 20 .

We have shown the PSNR curves for the four methods i.e. NeighShrink, ModiNeighShrink, NeighShrink (proposed) and ModiNeighShrink (proposed) in Figs. 3(a)-(c). It is evident from these figures that the PSNR gain curves of our method are good over the NeighShrink and ModiNeighShrink. We have applied our proposed scheme, NeighShrink, and ModiNeighShrink schemes for using different window sizes for noise level 10. The corresponding images are shown in Figs. 4(a)-(n). As evident from the denoised images given in Fig. 4, our proposed technique produces brighter and smoother denoised images than the NeighShrink and ModiNeighShrink. We have not shown the images for other two images, i.e., Barbara and Goldhill because of repetitive nature of results.

For low noise level, the denoising ability of our method gets worsened in case of large window size (refer Table 3 and corresponding Fig. 4(n) and Fig. 3(c)). On average we have found that neighbourhood window size of $3 \times 3$ is the better choice for our proposed method. From the above results and discussion, we have observed that our proposed schemes have better performance as compared to NeighShrink and ModiNeighShrink. We also got similar performance for two remaining images i.e. Barbara and Goldhill (refer Tables 1-3). But again because of repetitive nature, we have not shown them graphically.

Table 1. Denoising results (PSNR in db) for Lena, Barbra and Goldhill using window size of 3x3

\begin{tabular}{l|c|c|c|c|c}
\hline \multirow{4}{*}{ Image Name } & Noise levels & \multicolumn{2}{|c|}{ Used Threshold T1 } & \multicolumn{2}{c}{$\begin{array}{c}\text { Used Threshold T2 } \\
\text { (proposed) }\end{array}$} \\
\cline { 3 - 6 } & & NeiSh & MoNeiSh & NeiSh & MoNeiSh \\
\hline \multirow{5}{*}{ Lena } & 10 & 33.15 & 33.39 & 33.86 & 34.01 \\
\cline { 2 - 6 } & 20 & 28.46 & 28.79 & 29.76 & 30.05 \\
\cline { 2 - 6 } & 30 & 25.79 & 26.08 & 27.20 & 27.48 \\
\cline { 2 - 6 } & 35 & 24.92 & 25.18 & 26.31 & 26.58 \\
\cline { 2 - 6 } & 50 & 23.45 & 23.58 & 24.37 & 24.48 \\
\hline \multirow{5}{*}{ Barbara } & 10 & 30.99 & 31.34 & 31.84 & 32.09 \\
\cline { 2 - 6 } & 20 & 25.18 & 25.58 & 26.49 & 26.85 \\
\cline { 2 - 6 } & 30 & 22.49 & 22.72 & 23.49 & 23.80 \\
\cline { 2 - 6 } & 35 & 22.00 & 22.13 & 22.74 & 22.94 \\
\hline \multirow{5}{*}{ Goldhill } & 50 & 21.00 & 21.11 & 21.74 & 21.84 \\
\cline { 2 - 6 } & 20 & 30.59 & 30.90 & 31.48 & 31.71 \\
\cline { 2 - 6 } & 30 & 26.65 & 26.89 & 27.62 & 27.84 \\
\cline { 2 - 6 } & 35 & 24.75 & 24.95 & 25.79 & 26.00 \\
\cline { 2 - 6 } & 50 & 24.18 & 24.35 & 25.13 & 25.32 \\
\hline
\end{tabular}


Table 2. Denoising results (PSNR in db) for Lena, Barbra and Goldhill using window size of 5x5

\begin{tabular}{l|l|l|l|l|l}
\hline \multirow{3}{*}{ Image Name } & Noise levels & \multicolumn{2}{|c|}{ Used Threshold T1 } & \multicolumn{2}{c}{$\begin{array}{c}\text { Used Threshold T2 } \\
\text { proposed }\end{array}$} \\
\cline { 2 - 6 } & & NeiSh & MoNeiSh & NeiSh & MoNeiSh \\
\hline \multirow{5}{*}{ Lena } & 10 & 34.03 & 33.84 & 32.87 & 32.11 \\
\cline { 2 - 6 } & 20 & 30.24 & 30.49 & 30.93 & 30.90 \\
\cline { 2 - 6 } & 30 & 27.49 & 27.79 & 28.75 & 28.95 \\
\cline { 2 - 6 } & 35 & 26.54 & 26.80 & 27.79 & 28.04 \\
\cline { 2 - 6 } & 50 & 24.41 & 24.66 & 25.81 & 26.02 \\
\hline \multirow{5}{*}{ Barbara } & 10 & 32.44 & 32.39 & 32.06 & 31.64 \\
\cline { 2 - 6 } & 20 & 27.56 & 27.86 & 28.38 & 28.51 \\
\cline { 2 - 6 } & 30 & 24.41 & 24.75 & 25.62 & 25.93 \\
\cline { 2 - 6 } & 35 & 23.33 & 23.67 & 24.52 & 24.82 \\
\hline \multirow{5}{*}{ Goldhill } & 50 & 21.76 & 21.91 & 22.48 & 22.66 \\
\cline { 2 - 6 } & 20 & 32.16 & 32.10 & 31.62 & 31.15 \\
\cline { 2 - 6 } & 30 & 28.14 & 28.39 & 28.91 & 28.98 \\
\cline { 2 - 6 } & 35 & 26.08 & 26.30 & 26.99 & 27.16 \\
\cline { 2 - 6 } & 50 & 25.30 & 25.54 & 26.32 & 26.50 \\
\hline
\end{tabular}

Table 3. Denoising results (PSNR in db) for Lena, Barbra and Goldhill using window size of 7x7

\begin{tabular}{c|c|c|c|c|c}
\hline Image Name & Noise levels & \multicolumn{2}{|c|}{ Used Threshold T1 } & \multicolumn{2}{c}{$\begin{array}{r}\text { Used Threshold T2 } \\
\text { (proposed) }\end{array}$} \\
\cline { 3 - 6 } & & NeiSh & MoNeiSh & NeiSh & MoNeiSh \\
\hline \multirow{4}{*}{ Lena } & 10 & 30.71 & 30.05 & 29.39 & 28.99 \\
\cline { 2 - 6 } & 20 & 30.21 & 30.07 & 29.01 & 27.82 \\
\cline { 2 - 6 } & 30 & 28.24 & 28.45 & 28.64 & 28.47 \\
\cline { 2 - 6 } & 35 & 27.37 & 27.61 & 27.97 & 28.02 \\
\hline \multirow{5}{*}{ Barbara } & 50 & 25.20 & 25.48 & 26.08 & 26.30 \\
\cline { 2 - 6 } & 10 & 30.67 & 30.12 & 29.73 & 29.32 \\
\cline { 2 - 6 } & 20 & 28.32 & 28.30 & 27.83 & 27.10 \\
\cline { 2 - 6 } & 30 & 25.80 & 26.08 & 26.32 & 26.30 \\
\hline \multirow{5}{*}{ Goldhill } & 35 & 24.68 & 25.03 & 25.48 & 25.62 \\
\cline { 2 - 6 } & 50 & 22.45 & 22.72 & 23.23 & 23.52 \\
\cline { 2 - 6 } & 10 & 30.30 & 29.80 & 29.35 & 29.02 \\
\cline { 2 - 6 } & 20 & 28.79 & 28.73 & 28.01 & 27.09 \\
\cline { 2 - 6 } & 30 & 26.83 & 27.03 & 27.28 & 27.20 \\
\hline & 35 & 26.11 & 26.34 & 26.67 & 26.75 \\
\hline
\end{tabular}

Here, NeiSh and MoNeiSh represent NeighShrink and ModiNeighShrink methods respectively. 


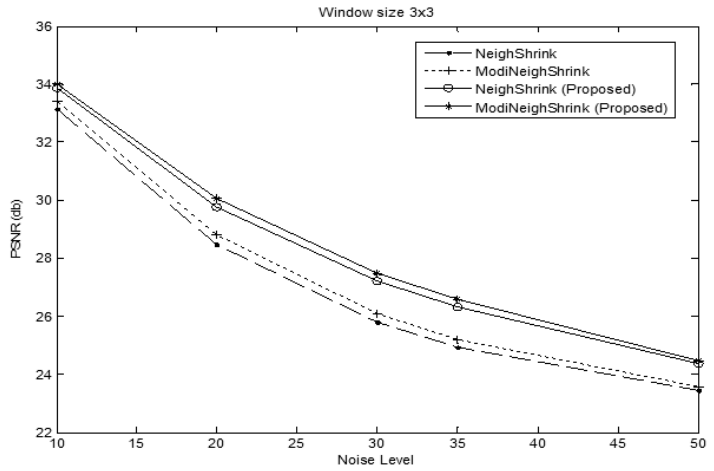

(a)

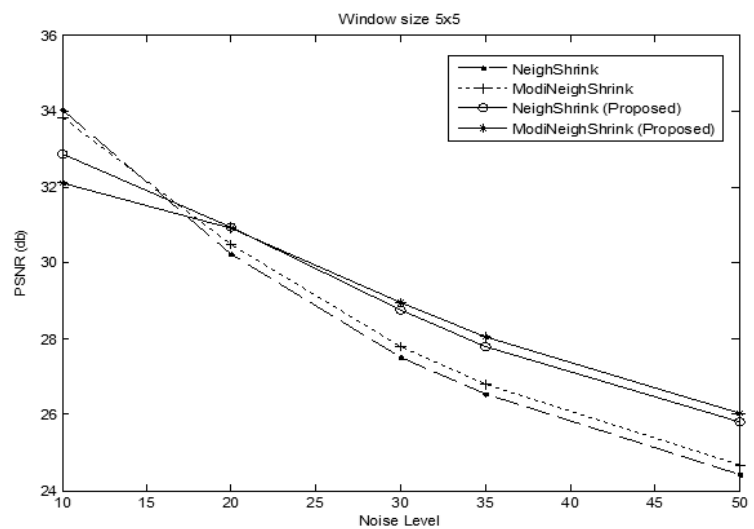

(b)

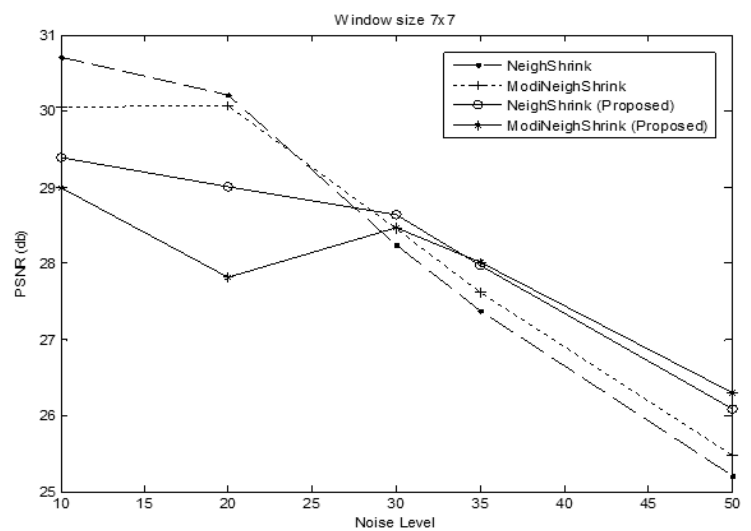

(c)

Figure 3. PSNR vs. Noise level of NeighShrink (proposed), ModiNeighShrink (proposed), NeighShrink, and ModineighShrink methods for Lena image with window size: (a) 3x3 (b) 5x5 and (c) 7x7 


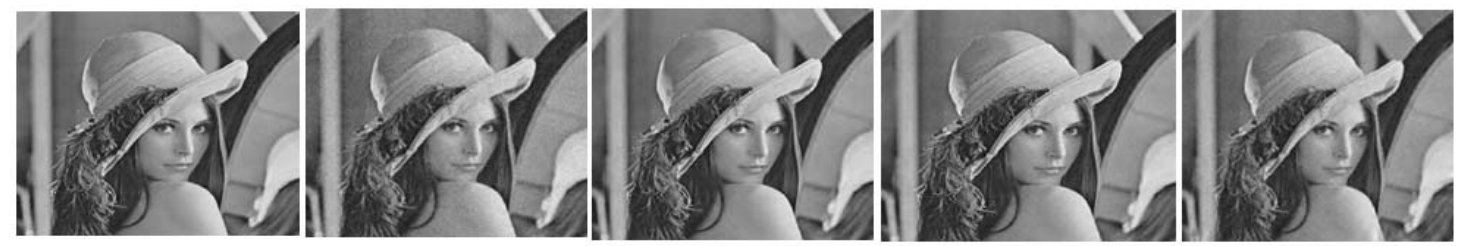

(a)

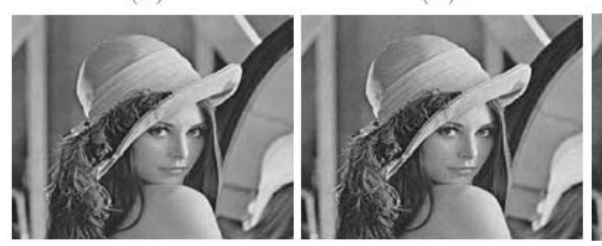

(f)

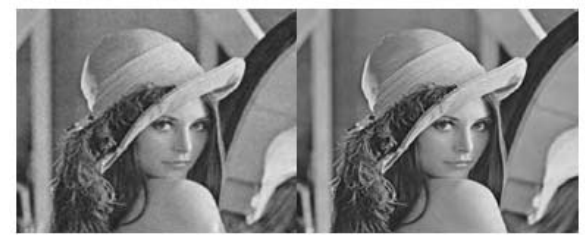

(k)
(1) (c)

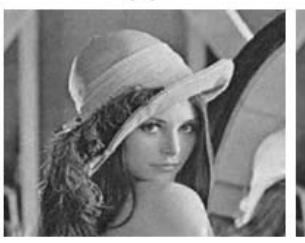

(h)

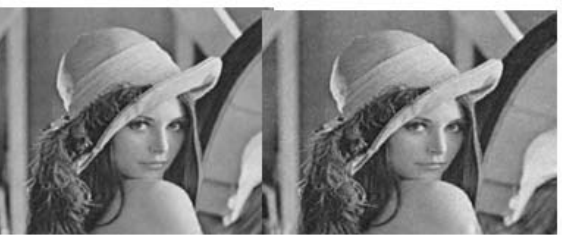

(iii) (d)

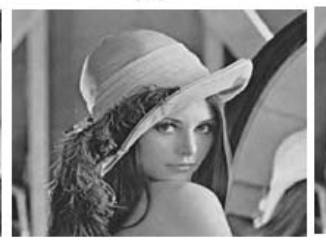

(i)

(n) (e)

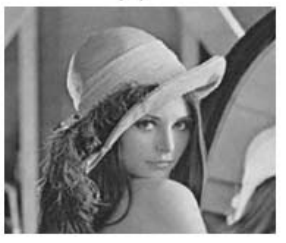

(j)

Figure 4. Original Lena image, its corrupted version with noise level 10 and denoised Lena image using different methods (a) Original (b) Noisy image with noise level 10 (c) Denoise using NeighShrink (3x3) (d) Denoise using NeighShrink (5x5) (e) Denoise using NeighShrink (7x7) (f) Denoise using ModiNeighShrink (3x3) (g) Denoise using ModiNeighShrink (5x5) (h) Denoise using ModiNeighShrink (7x7) (i) Denoise Using NeighShrink (Proposed) (3x3) (j) Denoise using NeighShrink (Proposed) (5x5) (k) Denoise using NeighShrink (Proposed) (7x7) (l) Denoise using ModiNeighShrink (Proposed) (3x3) (m) Denoise using ModiNeighShrink (Proposed) (5x5) and (n) Denoise using ModiNeighShrink (Proposed) (7x7).

\section{Conclusion}

In this paper, we have proposed a new scheme namely A Hybrid Image Denoising Technique Using Neighbouring Wavelet Coefficient for image denoising. Our method provides better results than the NeighShrink and ModiNeighShrink methods as far as visual quality of the image is concerned. We have also found that the window size $3 \times 3$ is the best choice for our methods. We have also obtained that the PSNR gained in our method is substantially higher than NeighShrink and ModiNeighShrink in experimental results.

Note: The proposed algorithm is implemented in MatLab Toolbox 7.5.0, running on a 3.20-GHz Pentium IV personal computer with 504 MB of RAM.

\section{References}

1. C. S. Burrus, R. A. Gopinath and H. Guo, "Introduction to wavelet and wavelet transforms," Prentice Hall, 1997.

2. M. Jansen, "Noise Reduction by Wavelet Thresholding," Springer - Verlag New York Inc., 2001.

3. Antoniadis and J. Bigot, "Wavelet Estimators in Nonparametric Regression: A Comparative Simulation Study," Journal of Statistical Software, Vol. 6, Issue 6, pp. 1-83, 2001.

4. D. L. Donoho and I. M. Johnstone, "Wavelet shrinkage: Asymptotic?" J.R. Stat. Soc. B, Vol. 57, No. 2, pp. 301369, 1995.

5. D. L. Donoho, "De-Noising by Soft Thresholding," IEEE Trans. Info., Vol. 41, No. 3, pp. 613-627, 1995. 
6. D. L. Donoho and I. M. Johnstone, "Adapting to Unknown Smoothness via Wavelet Shrinkage," Journal of American Statistical Association, Vol. 90, No. 432, pp. 1200-1224, 1995.

7. D. L. Donoho and I. M. Johnstone, "Ideal spatial adaptation via wavelet shrinkage," Biometrika, Vol. 81, No. 3, pp. 425-455, 1994.

8. X.P. Zhang and M. D. Desai, "Adaptive denoising based on SURE risk," IEEE Signal Process. Lett., Vol. 5, No. 10, pp. 265-267, 1998.

9. S. G. Chang, B. Yu and M. Vetterli, "Adaptive Wavelet Thresholding for Image Denoising and Compression," IEEE Trans. Image Processing, Vol. 9, No. 9, pp. 1532-1546, 2000.

10.Elyasi and S. Zarmehi, "Elimination Noise by Adaptive Wavelet Threshold," World Academy of Science, Engineering and Technology, pp. 462-466, 2009.

11.T. T. Cai and B.W. Silverman, "Incorporating information on neighboring coefficients into wavelet estimation," Sankhya, Ser. B, Vol. 63, No. 2, pp. 127-148, 2001.

12.G. Y. Chen, T. D. Bui and A. Krzy'zak, "Image denoising with neighbor dependency and customized wavelet and threshold," Pattern Recognition, Vol. 38, No. 1, pp. 115-124, 2005.

13.G.Y. Chen, T. D. Bui and A. Krzyzak, "Image denoising using neighbouring wavelet coefficients," ICASSP, pp. 917-920, 2004

14.S. K. Mohideen, S. A. Perumal and M. M. Sathik, "Image De-noising using Discrete Wavelet transform," IJCSNS International Journal of Computer Science and Network Security, Vol. 8, No. 1, pp. 213-216, 2008.

15.P. Kittisuwan and W. Asdornwised, "Wavelet-Based Image Denoising using NeighShrink and BiShrink Threshold Functions," ECTI-CON, pp. 497-500, 2008.

16.Daubechies, "Ten Lectures on Wavelets," Proc. CBMS-NSF Regional Conference Series in Applied Mathematics. Philadelphia, PA: SIAM, Vol. 61, 1992.

17.B. C. Rao1 and M. M. Latha, "Selective neighbouring wavelet coefficients approach for image denoising," International Journal of Computer Science and Communication, Vol. 2, No. 1, pp. 73-77, 2011.

18.G. Chen and W. Zhu, "Image Denoising Using Three Scales of Wavelet Coefficients," Advances in Neural Networks, Vol. 5264, pp. 376-383, 2008.

19.S. Gupta and L. Kaur, "Wavelet Based Image Compression using Daubechies Filters," In proc. 8th National conference on communications, I.I.T. Bombay, NCC, 2002.

20.Y. Yang and Y. Wei, "Neighboring Coefficients Preservation for Signal Denoising," Circuits, Systems, and Signal Processing, Vol. 31, No. 2, pp. 827-832, 2012.

21.Divya Guglani and Nitin Kumar Katyal, "Noise Removal using Double Density Complex Dual Tree Transform with NeighShrink SURE and Median Filter," 1st International Conference on Next Generation Computing Technologies, pp. 1-4, 2015. 